\title{
Mise au point
}

\section{Tuméfactions cervico-faciales per et post-opératoires immédiates en chirurgie bucco-dentaire}

\author{
Bartholomeus Calon ${ }^{1,3,}{ }^{*}$, Caroline Schellenberger ${ }^{2,3}$, Catherine-Isabelle Gros ${ }^{2,3}$, \\ Fabien Bornert ${ }^{2,3}$, Pierre Olivier Ludes ${ }^{1,3}$, Ahmed Féki ${ }^{2,3}$ \\ 1 UF d'Anesthésiologie-Réanimation, Hôpital de Hautepierre, Strasbourg, France \\ 2 UF de Chirurgie buccale et Implantologie, Pôle de Médecine et Chirurgie buccales, Hôpital Civil, Strasbourg, France \\ 3 Hôpitaux Universitaires, Strasbourg, France
}

\author{
(Reçu le 11 juin 2013, accepté le 10 juillet 2013)
}

Mots clés : tuméfaction cervico-faciale / dent / emphysème / hématome / angiœdème / fasciite

Key words: cervicofacial swelling / tooth / emphysema / hematoma / angioedema / fasciitis

Résumé - Il n'est pas rare que le chirurgien-dentiste soit confronté à une tuméfaction cervico-faciale (TCF) per ou post-opératoire immédiate. Bien qu'il s'agisse généralement d'un phénomène bénin, il est souvent source d'inquiétude pour le praticien et le patient. Une revue de littérature se propose de présenter les différentes étiologies de tuméfactions cervico-faciales per et post-opératoires, et d'apporter les éléments pratiques pour leur diagnostic. Parmi ces tuméfactions, l'emphysème sous-cutané est un évènement banal, bénin et facile à diagnostiquer mais souvent méconnu, ce dont témoigne le nombre de cas rapportés. Plus rares, mais potentiellement graves, il convient également de citer l'hématome du plancher buccal et l'angiœdème qui peuvent être la conséquence du geste bucco-dentaire ou des médicaments. Dans ce cadre, il est en effet nécessaire de savoir reconnaitre les prodromes d'une détresse respiratoire. Enfin, la fasciite nécrosante cervico-faciale se doit d'être reconnue tôt du fait de son extrême gravité, d'autant que son incidence paraît croissante. Un tableau récapitulatif présente les principales causes de TCF per et post-opératoires après chirurgie dentaire, leur chronologie, leurs principaux symptômes et les mesures thérapeutiques à mettre en œuvre.

\begin{abstract}
Cervicofacial swelling after dental treatment: what the dentist should know. Although generally harmless, the occurrence of cervicofacial swelling (CFS) during or after dental surgery can be worrisome for the dentist or the patient. This article is a literature review of most causes of CFS after dental surgery. Practical diagnosis tools should allow distinguishing between benign and dangerous situations. Indeed, the occurrence of a spectacular but harmless condition like subcutaneous emphysema may cause unnecessary concern, as witnessed by the many case reports in medical literature. On the other hand, conditions like hematoma of the floor of the mouth or angioedema may be caused by the dentist (through surgery or medication) and can become lifethreatening. Upper airway compromise should be recognized early on. Cervical fasciitis (CF) is a severe and often fulminating septic emergency with potentially dramatic consequences, warranting immediate intensive and surgical care. The increasing incidence of CF and its possible occurrence after dental surgery warrants a high level of suspicion in a septic patient. Finally, a summary chart will point out the time course, symptoms and management of the different causes of CFS after dental surgery.
\end{abstract}

Lors d'un acte de chirurgie bucco-dentaire ou dans son décours immédiat, la survenue d'une tuméfaction cervicofaciale (TCF) n'est pas rare. Si elle présente généralement un aspect bénin (bien qu'inquiétant parfois pour le praticien ou le patient), elle peut en de rares occasions, nécessiter une prise en charge spécifique. Le but de ce travail était de faire une revue synthétique des éléments cliniques associés aux différentes causes des TCF survenant après un acte de chirurgie bucco-dentaire. Ces éléments permettront d'en affirmer le caractère bénin ou, au contraire, de suspecter une éventuelle urgence médicale. Les différentes pathologies seront exposées et un tableau récapitulatif permettra une visualisation rapide de ces pathologies en fonction de leurs délais d'apparition, des signes associés et des gestes à réaliser le cas échéant.

\footnotetext{
*Correspondance : Bartholomeus.Calon@chru-strasbourg.fr
} 


\section{Étiologies}

Les TCF per ou post-opératoires immédiates (dans les $48 \mathrm{~h}$ qui suivent l'intervention) en chirurgie bucco-dentaire sont caractérisées par la survenue inopinée d'un gonflement facial et/ou cervical chez un patient préalablement asymptomatique. Elles ont des caractéristiques différentes en fonction de leur origine et leur présentation est faite selon leur délai de survenue.

\section{Extrusion d'hypochlorite de sodium ( $\mathrm{NaOCl})$ [1]}

Le $\mathrm{NaOCl}$ est utilisé comme irrigant endodontique pour son pouvoir bactéricide et ses capacités de dissolution des tissus nécrotiques (composants organiques dentinaires). Sa toxicité, qui augmente avec sa concentration, est liée à l'alcalinité et l'hypertonicité de la solution, ainsi qu'à la production de soude et d'hypochlorure $\left(\mathrm{NaOCl}+\mathrm{H}_{2} \mathrm{O} \rightarrow \mathrm{NaOH}+\mathrm{HClO}\right)$.

L'extrusion d'hypochlorite au-delà de l'apex radiculaire peut être à l'origine de :

- brûlures chimiques : elles conduisent à des nécroses tissulaires localisées ou extensives, avec une tuméfaction inflammatoire ; celle-ci est œdémateuse et/ou hémorragique ; et dans le sinus maxillaire, elle peut provoquer une sinusite aiguë [2].

- propagation de l'infection : en présence d'une lésion périapicale, des bactéries canalaires peuvent être portées dans les tissus sains périphériques entraînant un risque d'infection secondaire ;

- complications neurologiques : il s'agit de paresthésies et d'anesthésie trigéminales (branches mentonnière, alvéolaire inférieure ou infra-orbitaire), et/ou d'atteinte motrice (parésie de la branche buccale du nerf facial) ; la récupération, qu'elle soit sensitive ou motrice, peut prendre plusieurs mois ;

- obstruction des voies aériennes supérieures : celle-ci peut résulter d'une atteinte directe de la muqueuse, par fuite du produit dans la cavité buccale, mais également d'un hématome extensif et compressif du plancher buccal, pouvant mettre en jeu le pronostic vital [3] ;

- allergies : le $\mathrm{NaOCl}$ pourrait être responsable d'une allergie de type anaphylactique [1].

La symptomatologie est habituellement spectaculaire, caractérisée par une douleur intense et immédiate, associée à une tuméfaction. L'œdème inflammatoire et l'hématome apparaissent généralement dans les minutes qui suivent. Mais la douleur et la tuméfaction sont parfois retardées de plusieurs heures. L'apparition d'une dysphonie, d'une dyspnée, d'un trismus et d'une sialorrhée doit faire évoquer une obstruction imminente des voies aériennes supérieures.
Le traitement initial comprend : irrigation au sérum physiologique, analgésie (froid, paracétamol, anti-inflammatoires non stéroïdiens), corticoïdes et antibiotiques. La survenue de signes d'obstruction des voies aériennes impose une surveillance en milieu spécialisé dans l'éventualité d'un geste de décompression.

\section{Emphysème sous-cutané [4]}

L'emphysème sous-cutané (ESC), dans les suites immédiates d'un acte bucco-dentaire, représenterait un incident banal s'il n'était pas trop souvent méconnu et source d'inquiétude pour le praticien et le patient [5]. De nombreux cas sont rapportés [4]. Des complications gravissimes lui sont souvent imputées mais une revue exhaustive de la littérature ne permet pas de retrouver de morbidité significative ou de mortalité liées à un ESC. Par conséquent, il est important de savoir le reconnaître pour éviter de le dramatiser.

Après un acte bucco-dentaire, il est dû à la propagation d'air sous pression dans les tissus sous-cutanés, à partir d'une lésion de l'alvéole dentaire, des canaux radiculaires ou d'une lésion muqueuse.

L'ESC peut être provoqué :

- par le praticien : l'ESC survient de façon immédiate ou rapide ; il est généralement favorisé par l'utilisation d'une turbine à air, d'une pièce à main à haute vitesse, d'un spray air/eau, d'une seringue pour l'irrigation canalaire ou d'un laser équipé d'un système de projection d'air ;

- par le patient : il s'agit d'une éventualité rare due à une pression intra-orale créée par le patient; la description princeps a été faite chez un joueur de trompette après avulsion dentaire. On peut encore citer le fait de se moucher, de tousser ou même de gonfler un ballon (Fig. 1) [6]. Contrairement à l'ESC iatrogène, sa survenue se fait à distance de l'acte dentaire, au moment de l'hyperpression.

Il se traduit par l'apparition rapide d'une tuméfaction des tissus mous de la face et du cou, avec extension possible vers la région thoracique. C'est une lésion indolore et non érythémateuse, il n'existe ni gêne ni dyspnée, la voix peut en revanche être nasonnée. À la palpation, on retrouve une crépitation neigeuse pathognomonique.

Le diagnostic clinique est facile : il ne devrait pas être nécessaire de recourir à des examens complémentaires (radiographies, scanner, IRM ou biopsie) [5] pour rassurer le patient. La régression se fait en moins de 5 jours, sans mesures particulières (Figs. 2 et 3 ). Une antibiothérapie n'est pas préconisée [4] mais elle est souvent prescrite. L'extension thoracique de l'ESC justifie d'une surveillance en milieu hospitalier bien que la survenue d'un pneumomédiastin ou d'un pneumothorax compressif reste théorique. 


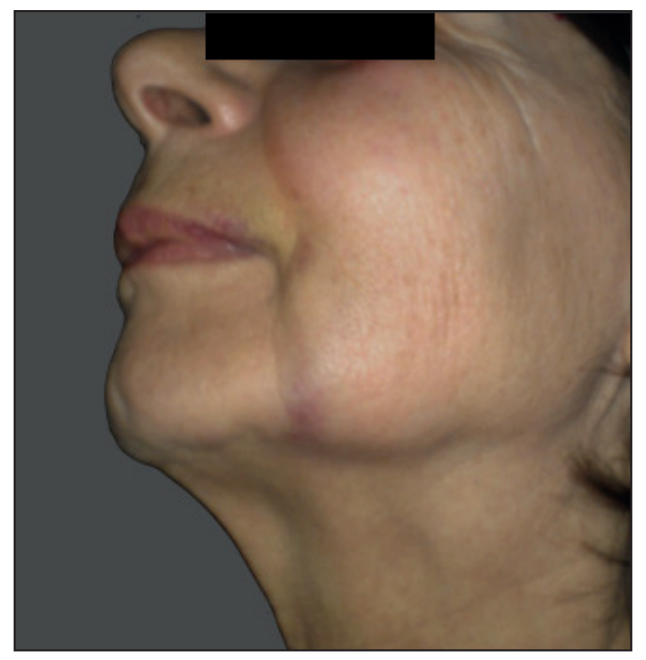

Fig. 1. Emphysème sous-cutané apparu $1 \mathrm{~h}$ après un acte de chirurgie bucco-dentaire, en gonflant un ballon.

Fig. 1. Subcutaneous emphysema one hour after dental surgery while blowing up a balloon.

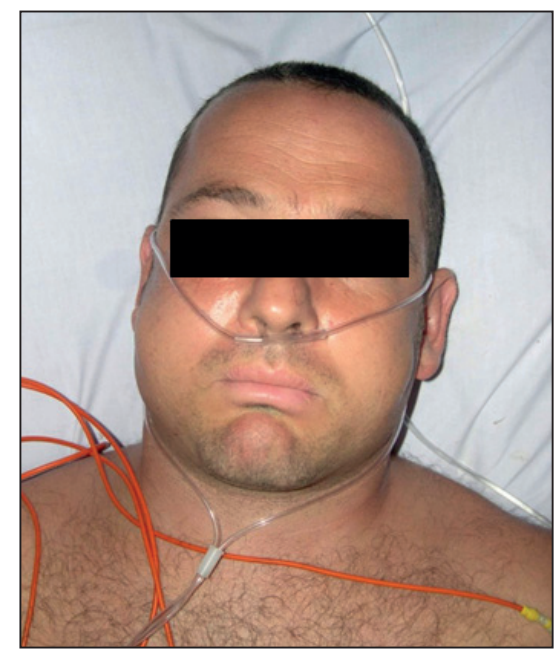

Fig. 2. Emphysème sous-cutané cervico-facial diffus post-traumatique. Fig. 2. Diffuse subcutaneous post-traumatic cervicofacial emphysema.

\section{Angiœdème [7-9]}

Le terme " angiœdème » $(\mathrm{A} 0)$ recouvre un ensemble de pathologies multifactorielles dont l'expression clinique est commune. L'A0 est caractérisé par la survenue soudaine d'œdèmes hypodermiques sous-cutanés ou sous-muqueux, localisés et transitoires.

Ces œdèmes sont provoqués par l'histamine, les leucotriènes ou la bradykinine qui entraînent une vasodilatation avec une perméabilité capillaire et post-capillaire augmentée.

- L'histamino-libération mastocytaire cutanéo-muqueuse peut résulter d'un mécanisme allergique spécifique IgE-dépendant

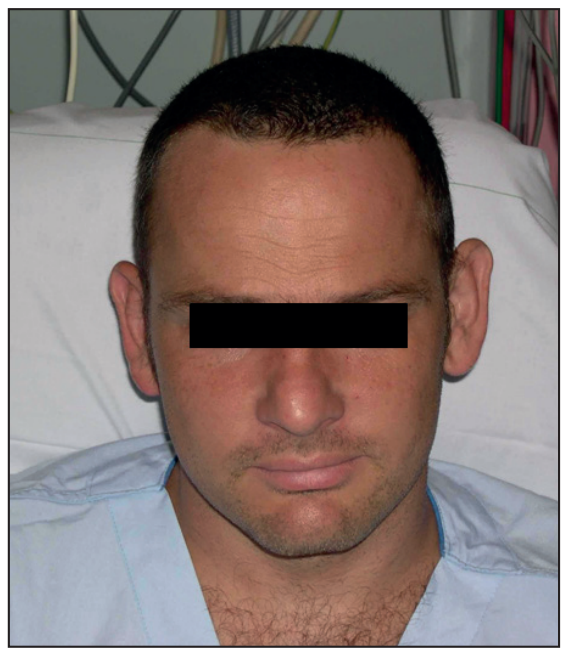

Fig. 3. Même patient après quelques jours, régression spontanée de l'emphysème sous-cutané.

Fig. 3. Same patient, spontaneous regression of subcutaneous emphysema within a few days.

(aliments, médicaments, venins d'hyménoptères) ou non allergique (histamino-libération non spécifique d'origines physique, alimentaire ou médicamenteuse). Au cabinet dentaire, l'histamino-libération spécifique peut être provoquée par les ß-lactamines ou le latex, et l'histamino-libération non spécifique par la pression ou les morphinomimétiques par exemple.

- Les leucotriènes, produits à partir d'une altération du métabolisme de l'acide arachidonique, sont à l'origine de l'intolérance aux anti-inflammatoires non stéroïdiens (AINS), à l'acide salicylique et aux sulfites.

- La bradykinine peut être à l'origine d'A0 par excès de synthèse ou défaut de dégradation des kinines.

Les crises peuvent être déclenchées par un traumatisme chirurgical (extraction dentaire ...). On distingue les :

- A0 héréditaires (déficit quantitatif ou qualitatif en inhibiteur de la (1 estérase) ;

- A0 acquis (déficit quantitatif en inhibiteur de la C1 estérase) ;

- A0 de type III (sensibilité aux estrogènes) ;

- A0 iatrogènes (médicaments inhibiteurs de l'enzyme de conversion, antagonistes des récepteurs de l'angiotensine II, inhibiteurs du dipeptidyl peptidase 4) [10].

Les $\mathrm{A} 0$ touchent les zones cutanées lâches et extensibles telles que la face, les mains et les organes génitaux externes, ainsi que l'intestin. Il n'est pas douloureux et ne prend pas le godet mais sa gravité réside dans l'obstruction possible des voies aériennes supérieures par atteinte de la muqueuse 
Tableau I. Thérapeutiques de l'A0 en fonction de la gravité (d'après [7, 9-14]). Table I. Angioedema treatment according to severity (as in [7, 9-14]).

\begin{tabular}{|c|c|c|c|}
\hline & $\begin{array}{l}\text { Asphyxie : } \\
\quad \text { traitement d'urgence }\left(+\mathrm{O}_{2}\right)\end{array}$ & $\begin{array}{l}\text { Formes sans gravité : } \\
\quad \text { traitement }\end{array}$ & Traitement de fond \\
\hline A0 histaminique & $\begin{array}{l}\text { Salbutamol } \\
\quad \text { - spray }(2,5-7,5 \mathrm{mg}) \\
\text { Adrénaline IM : } \\
\quad \text { - adulte }: 0,3-0,6 \mathrm{mg} \\
\quad \text { - enfant }: 0,15 \mathrm{mg}\end{array}$ & $\begin{array}{l}\text { Corticothérapie par voie générale } \\
\text { Antihistaminiques } \mathrm{H} 1 \text { et } \mathrm{H} 2\end{array}$ & $\begin{array}{l}\text { Eviction de l'allergène } \\
\text { Antihistaminiques }\end{array}$ \\
\hline $\begin{array}{l}\text { A0 lié aux } \\
\text { leucotriènes }\end{array}$ & $\begin{array}{l}\text { Corticoïdes } \\
\quad \text { - prednisone } 1 \mathrm{mg} / \mathrm{kg}\end{array}$ & Corticoïdes & $\begin{array}{l}\text { Antagonistes des récepteurs } \\
\text { des leucotriènes }\end{array}$ \\
\hline A0 bradykinique & $\begin{array}{l}\text { Intubation trachéale } \\
\text { Inhibiteur de la C1 estérase IV } \\
\text { Icatibant IC }\end{array}$ & $\begin{array}{l}\text { Acide tranexamique per os ou IV } \\
(1 \mathrm{~g} \text { ou } 10 \mathrm{mg} / \mathrm{kg} \text { chez l'enfant) }\end{array}$ & $\begin{array}{l}\text { Eviction des médicaments } \\
\text { responsables } \\
\text { Acide tranexamique, danazol }\end{array}$ \\
\hline
\end{tabular}

pharyngo-laryngée. Devant un A0 de la face, il faut rechercher d'une part les signes de gravité (dysphonie, dyspnée inspiratoire, hypersalivation, œdème de la luette) et, d'autre part, les signes orientant vers l'étiologie (symptômes associés, délais) puisque la prise en charge en dépend.

- Ainsi, les AO histaminiques sont d'aspect « rouge » (érythème périphérique, urticaire, rhino-conjonctivite, prurit) ; leur délai d'apparition est bref (quelques min à $2 \mathrm{~h}$, en fonction de la voie d'administration de l'allergène) et leur durée limitée $(<12-24 \mathrm{~h})$; ils peuvent être associés à une crise d'asthme ou un choc anaphylactique.

- Les A0 liés aux leucotriènes se présentent soit sous une forme cutanéo-muqueuse, d'aspect inflammatoire avec urticaire, rhinite et conjonctivite ou sous une forme respiratoire, avec asthme dans le cadre d'une polypose nasale (syndrome de Fernand Widal). L'AO apparait plus tardivement (4-24 $\mathrm{h}$ après prise d'AINS), alors que l'asthme, la rhinite et la conjonctivite peuvent survenir plus tôt (30120 min après la prise d'AINS) [11].

- Les A0 bradykiniques ne sont pas prurigineux ni inflammatoires. La forme ORL peut être précédée de formes abdominales. Sa survenue est plus tardive (quelques $h$ ) et sa durée prolongée (24-96 h).

Le chirurgien dentiste peut donc être confronté à un $\mathrm{A} 0$ dans des circonstances multiples, au cabinet ou dans les suites d'un acte bucco-dentaire. Il lui appartient surtout de rechercher les éléments de gravité (les « 3 dys »: dysphonie, dysphagie, dyspnée) et de diagnostic (présence ou absence d'érythème, d'urticaire) pour les transmettre le cas échéant au SAMU (téléphone : 15 ou 112), ce qui permettra au médecin de fournir les indications thérapeutiques d'urgence (Tab. I). Les formes graves, asphyxiantes des AO histaminiques justifient l'utilisation d'un spray de salbutamol ou d'une injection intra-musculaire d'adrénaline. Au contraire, les A0 bradykiniques ne sont généralement pas sensibles à l'adrénaline ni aux corticoïdes [10] et leur traitement repose sur l'administration intra-veineuse de concentré d'inhibiteur de la C1 estérase ou d'antagoniste des récepteurs B2 bradykiniques (icatibant en injection sous-cutanée) ; l'intubation trachéale peut s'avérer nécessaire. La place du concentré de complexe prothrombinique reste à définir [10].

Les A0 liés aux leucotriènes réagissent au traitement par les corticoïdes.

L'administration d'oxygène (10-15 L/min) est licite dans tous les cas, ainsi que la position semi-assise en l'absence d'état de choc.

\section{Avulsion dentaire [15-17]}

La chirurgie bucco-dentaire peut s'accompagner d'une tuméfaction post-opératoire, essentiellement après avulsion des dents postérieures. Il s'agit d'une réaction inflammatoire post-traumatique aiguë avec libération locale de médiateurs de l'inflammation (histamine, sérotonine, bradykinine, prostaglandines) entraînant une vasodilatation et une augmentation de la perméabilité vasculaire.

Il s'agit d'une tuméfaction unilatérale, non extensive, apparaissant immédiatement après l'intervention avec un maximum $d^{\prime}$ intensité situé entre la $48^{\mathrm{e}}$ et la $72^{\mathrm{e}} \mathrm{h}$, et une régression spontanée vers le $5-7^{\mathrm{e}}$ jour post-opératoire. Elle est souvent accompagnée de douleurs et d'un trismus. La tuméfaction est douloureuse à la palpation mais ne prend pas le godet; à l'examen endo-buccal, on observe un œdème de la muqueuse environnante. Il n'existe pas de signes locaux (dysphonie, dyspnée) ni généraux (fièvre, sepsis) inquiétants. 
Le traitement est médicamenteux pré et/ou post-opératoire (AINS, corticoïdes, enzymes) et non médicamenteux (cryothérapie, laser) [17].

\section{Hématome du plancher buccal $[18,19]$}

La richesse de la vascularisation de la corticale linguale de la mandibule dans la région inter-foraminale explique la survenue éventuelle d'un hématome sublingual lors d'un acte bucco-dentaire entraînant une perforation de la corticale. Les tissus lâches du plancher buccal permettent l'extension sousmentale, sublinguale et sous-mandibulaire de l'hématome, ce qui peut aboutir à une obstruction des voies aériennes supérieures par élévation du massif lingual vers la voute palatine. Cette complication s'observe le souvent lors d'une intervention chirurgicale dans la région canine. La prise d'anticoagulants ou d'antiagrégants plaquettaires peut constituer un facteur favorisant.

La survenue de l'hématome est le plus souvent immédiate, plus rarement tardive, probablement en raison de la vasoconstriction initiale liée à l'anesthésie locale [20]. Les symptômes cliniques comportent l'apparition d'un gonflement hémorragique du plancher buccal avec protrusion linguale, dysphonie et détresse respiratoire.

L'urgence réside dans le contrôle des voies aériennes supérieures : en cas de détresse respiratoire, il faut procéder à une intubation trachéale (sous fibroscopie vigile éventuellement) ou à une trachéotomie.

L'hémostase initiale est assurée par compression mandibulaire bi-manuelle lors du transport du patient. Secondairement, on réalise l'hémostase par ligature artérielle extra ou intraorale et l'évacuation de l'hématome. La radiologie interventionnelle, avec mise en place de spires métalliques ou de particules non résorbables dans le territoire lésé, peut éviter une intervention chirurgicale rendue délicate par l'importance des remaniements lié à l'hématome [21]. Souvent, le contrôle des voies aériennes est suffisant pour passer la période critique car l'extension de l'hématome prend souvent fin rapidement.

Il est important de connaître la possibilité d'une survenue tardive de l'hématome ; l'appel téléphonique doit déclencher une prise en charge rapide en cas de détresse respiratoire imminente.

\section{Cellulite cervico-faciale $[22,23]$}

Les cellulites cervico-faciales odontogènes sont des infections qui présentent un développement trans-ostéo-périosté permettant la diffusion vers les tissus cellulo-graisseux et musculaires profonds de la face et du cou. Elles peuvent survenir après un acte de chirurgie bucco-dentaire $(0,8 \%$ après extraction de $3^{\mathrm{e}}$ molaire mandibulaire) [24]. En général il n'existe pas de facteurs favorisants connus mais l'alcoolisme et le tabagisme sont des facteurs fréquemment associés.
La responsabilité des anti-inflammatoires non stéroïdiens (AINS) a été souvent évoquée mais elle n'a jamais été démontrée [25]. Le plus souvent circonscrites et bénignes, leur gravité réside dans les évolutions diffuses, inopinées et potentiellement malignes (mortalité de $7 \%$, durée d'hospitalisation moyenne de 4 semaines) [25] :

- Cellulite bilatérale sous-maxillaire asphyxiante par refoulement supérieur et postérieur de la langue : c'est l'angine de Ludwig, très rare aujourd'hui.

- Fasciite nécrosante, toxi-infection extensive et rapide, voire fulgurante, des tissus mous avec atteinte des fascia, dont le pronostic est sévère ; son incidence semble croissante [25].

- Médiastinite, par extension médiastinale de la nécrose ; la responsabilité des corticoïdes est évoquée [26].

- Thrombophlébite jugulaire interne avec emboles septiques ou syndrome de Lemierre [27].

À l'examen bactériologique $[25,28]$, on retrouve surtout des streptocoques (groupes F, A et non groupables), des bacilles anaérobies gram négatif (Prevotella, Fusobacterium, Bacteroides), des cocci gram positif (Peptostreptococcus) et des staphylocoques. D'autres germes opportunistes peuvent être rencontrés chez le sujet immuno-compromis.

Les cellulites circonscrites post-opératoires ne surviennent généralement que quelques jours après le geste dentaire. La cellulite séreuse est purement inflammatoire (rougeur, chaleur, tumeur et douleur), alors qu'au stade suppuré les signes locaux (douleur pulsatile, trismus, dysphagie, tuméfaction fluctuante, adénopathies, hypersialorrhée et halitose) peuvent être associés à des signes généraux modérés (fièvre, asthénie).

Les cellulites diffuses graves apparaissent en moyenne après 11 jours d'évolution (de 2 à 60 jours) [25], mais elles peuvent se présenter sous une forme fulgurante avec fasciite nécrosante dans les 2 à $8 \mathrm{~h}$ post-opératoires [25,29]. Trismus, dysphagie, odynophagie, rougeur cervicale et fièvre sont les signes les plus fréquents. Douleur thoracique, rougeur pré-sternale et crépitation sous-cutanée sont moins fréquentes mais associées à un risque de médiastinite. La dyspnée et la dysphonie sont des signes plus rares. Douleurs lors de mouvements de la langue [30], écoulement de salive et paresthésies dans les territoires concernés [28] font évoquer une angine de Ludwig. Les signes généraux témoignant d'un sepsis sévère (fièvre élevée, altération rapide de l'état général, syndrome confusionnel) doivent inquiéter.

Devant l'apparition de signes locaux (douleur cervicale diffuse, trismus et odynophagie) associés à des signes généraux en faveur d'un sepsis sévère, il faut adresser rapidement le patient en milieu adapté pour permettre des mesures de réanimation spécialisée (déchocage) et la réalisation d'un scanner facio-cervico-thoracique avec injection de produit de contraste. 
Tableau II. Délai d'apparition, symptomatologie et prise en charge au cabinet dentaire des tuméfactions per et post-opératoires en fonction de leur étiologie.

Table II. Onset, symptoms and management in the dental office of per and postoperative swelling according to their etiology.

\begin{tabular}{|c|c|c|c|c|c|c|c|c|c|}
\hline \begin{tabular}{|l|} 
Étiologie \\
de l'oedème
\end{tabular} & $\begin{array}{c}\text { Délai } \\
\text { d'apparition }\end{array}$ & Douleurs & Godet & Crépitation & Stridor & Trismus & $\begin{array}{c}\text { Urticaire, } \\
\text { prurit, } \\
\text { vasodilatation }\end{array}$ & Inflammation & $\begin{array}{c}\text { Prise en charge en } \\
\text { urgence au cabinet } \\
\text { dentaire }\end{array}$ \\
\hline \begin{tabular}{|l|} 
Extrusion \\
d'hypochlorite
\end{tabular} & $0-5 \min$ & +++ & $\varnothing$ & $\varnothing$ & $\varnothing$ à ++ & $+/-$ & $\varnothing$ & $+/-$ & Glace \\
\hline $\begin{array}{l}\text { Emphysème } \\
\text { sous cutané }\end{array}$ & $0-60 \mathrm{~min}$ & $\varnothing$ & $\varnothing$ & ++ & $\varnothing$ & $\varnothing$ & $\varnothing$ & $\varnothing$ & Rassurer \\
\hline \begin{tabular}{|l} 
Angioedème \\
histaminique
\end{tabular} & $0-60 \mathrm{~min}$ & $\varnothing$ & $\varnothing$ & $\varnothing$ & + à ++ & $\varnothing$ & $+\grave{a ̀ ~++}$ & $\varnothing$ & $\begin{array}{l}\text { Adrénaline } \\
\text { SAMU }\end{array}$ \\
\hline $\begin{array}{l}\text { Avulsion(s) } \\
\text { (dents postérieures) }\end{array}$ & $60 \mathrm{~min}-24 \mathrm{~h}$ & + & $\varnothing$ & $\varnothing$ & $\varnothing$ & + & $\varnothing$ & $+/-$ & Symptomatique \\
\hline $\begin{array}{l}\text { Hématome } \\
\text { du plancher buccal }\end{array}$ & $60 \min -24 \mathrm{~h}$ & + & $\varnothing$ & $\varnothing$ & $\varnothing \mathrm{à} \mathrm{++}$ & $+/-$ & $\varnothing$ & $\varnothing$ & $\begin{array}{c}\text { Compression } \\
\text { SAMU }\end{array}$ \\
\hline Fasciite nécrosante & $2 \mathrm{~h}-48 \mathrm{~h}$ & ++ & $\varnothing$ & + & $+/-$ & + & $\varnothing$ & \begin{tabular}{|c} 
++ \\
bilatéral cervical \\
sepsis sévère
\end{tabular} & SAMU \\
\hline $\begin{array}{l}\text { Angioedème liè aux } \\
\text { leucotriènes (AINS) }\end{array}$ & $4 \mathrm{~h}-24 \mathrm{~h}$ & $\varnothing$ & $\varnothing$ & $\varnothing$ & + à ++ & $\varnothing$ & $\varnothing$ & $\varnothing$ & Corticoïdes \\
\hline \begin{tabular}{|l|} 
Angioedème \\
bradykinique
\end{tabular} & $12 \mathrm{~h}-48 \mathrm{~h}$ & $\varnothing$ & $\varnothing$ & $\varnothing$ & + à ++ & $\varnothing$ & $\varnothing$ & $\varnothing$ & SAMU \\
\hline Cellulite séreuse & $48 \mathrm{~h}$ & + & $\varnothing$ & $\varnothing$ & $\varnothing$ & $\varnothing$ & $\varnothing$ & + & $\begin{array}{l}\text { Trépanation / } \\
\text { extraction } \\
\text { Antibiotiques }\end{array}$ \\
\hline Cellulite suppurée & $48 \mathrm{~h}$ & ++ & + & $\varnothing$ & $\varnothing$ & + & $\varnothing$ & ++ & $\begin{array}{c}\text { Trépanation / } \\
\text { extraction } \\
+ \text { drainage } \\
\text { Antibiotiques }\end{array}$ \\
\hline
\end{tabular}

La perméabilité des voies aériennes est maintenue par intubation (éventuellement fibroscopique), voire par trachéotomie (1 fois sur 6) [25].

La porte d'entrée et les loges cervicales et thoracique envahies sont mises à plat chirurgicalement.

La prise en charge du sepsis comprend des mesures de réanimation et la prescription d'une association antibiotique comportant initialement une céphalosporine de $3^{\mathrm{e}}$ génération et un imidazolé (durée de $15 \mathrm{j}$ minimum), qui sera adaptée secondairement en fonction des résultats bactériologiques.

La place de l'oxygénothérapie hyperbare reste à définir.

\section{Conclusion}

La connaissance des délais de survenue et des symptômes des différentes causes de tuméfaction cervico-faciale après un acte de chirurgie bucco-dentaire doit permettre au chirurgiendentiste de reconnaître la plupart des causes bénignes tout en cherchant à éliminer les rares situations nécessitant un geste rapide ou un transfert urgent en milieu spécialisé.
Conflits d'intérêt : aucun

\section{Références}

1. Spencer HR, Ike V, Brennan PA. Review: the use of sodium hypochlorite in endodontics - potential complications and their management. Br Dent J 2007;202:555-9.

2. Kavanagh $\mathrm{CP}$, Taylor $\mathrm{J}$. Inadvertent injection of sodium hypochlorite into the maxillary sinus Br Dent J 1998;185:336337.

3. Bowden JR, Ethunandan M, Brennan PA. Life-threatening airway obstruction secondary to hypochlorite extrusion during root canal treatment. Oral Surg Oral Med Oral Pathol Oral Radiol Endod 2006;101:402-4.

4. McKenzie WS, Rosenberg M. Iatrogenic subcutaneous emphysema of dental and surgical origin: a literature review. J Oral Maxillofac Surg 2009;67:1265-8.

5. Frühauf J, Weinke R, Pilger U, Kerl H, Müllegger RR. Soft tissue cervicofacial emphysema after dental treatment: report of 2 cases with emphasis on the differential diagnosis of angioedema. Arch Dermatol 2005;141:1437-40. 
6. Maxwell MG, Thompson KM, Hedges MS. Airway compromise after dental extraction. J Emerg Med 2011;41:e39-41.

7. Barbara DW, Ronan KP, Maddox DE, Warner MA. Perioperative angioedema: background, diagnosis, and management. J Clin Anesth 2013 May 6. doi:ppi: S0952-8180(13)00118-9.

8. Bernstein JA, Moellman J. Emerging concepts in the diagnosis and treatment of patients with undifferentiated angioedema. Int J Emerg Med 2012;5:39.

9. Hosotte $M$, Jarlot $S$, Luyasu $S$, Kanny $G$. Oedème de Quincke ou angiœdèmes. Rev Prat 2012;62:242-4.

10. Millot I, Plancade D, Hosotte M, Landy C, Nadaud J, Ragot C, Graffin B, Drouet C, Kanny G. Treatment of a life-threatening laryngeal bradykinin angio-oedema precipitated by dipeptidylpeptidase-4 inhibitor and angiotensin-I converting enzyme inhibitor with prothrombin complex concentrates. $\mathrm{Br} J$ Anaesth 2012;109:827-9.

11. Leimgruber A. Allergo-immunologie. 1. Nouveautés dans le traitement des crises aiguës d'angiœdème héréditaire. Rev Med Suisse 2011;7:103-5.

12. Nosbaum A, Bouillet L, Floccard B, Javaud N, Launay D, Boccon-Gibod I, Fain 0, Groupe d'experts du CREAK. Prise en charge des angioœdèmes induits par les inhibiteurs de l'enzyme de conversion de l'angiotensine: recommandations du Centre de référence national des angiœdèmes. Rev Med Interne 2013;34: 209-13.

13. Dewachter P, Mouton-Faivre C, Nace L, Longrois D, Mertes P-M. Prise en charge d'une réaction anaphylactique en extrahospitalier et aux urgences: revue de la littérature. Ann Fr Anesth Reanim 2007;26:218-28.

14. Floccard B, Crozon J, Rimmelé T, Vulliez A, Coppere B, Chamouard V, Boccon-Gibod L, Bouillet L, Allaouchiche B. Prise en charge en urgence de l'angiœdème à bradykinine. Ann Fr Anesth Reanim 2011;30:578-88.

15. Klongnoi B, Kaewpradub P, Boonsiriseth K, Wongsirichat N. Effect of single dose preoperative intramuscular dexamethasone injection on lower impacted third molar surgery. Int J Oral Maxillofac Surg 2012;41:376-9.

16. Rana M, Gellrich N-C, Ghassemi A, Gerressen M, Riediger D, Modabber A. Three-dimensional evaluation of postoperative swelling after third molar surgery using 2 different cooling therapy methods: a randomized observer-blind prospective study. J Oral Maxillofac Surg 2011;69:2092-8.
17. Markovic A, Todorovic L. Effectiveness of dexamethasone and low-power laser in minimizing oedema after third molar surgery: a clinical trial. Int J Oral Maxillofac Surg 2007;36:226-9.

18. Pigadas N, Simoes P, Tuffin JR. Massive sublingual haematoma following osseo-integrated implant placement in the anterior mandible. Br Dent J 2009;206:67-8.

19. Kalpidis CDR, Setayesh RM. Hemorrhaging associated with endosseous implant placement in the anterior mandible: a review of the literature. J Periodontol 2004;75:631-45.

20. Dubois L, de Lange J, Baas E, Van Ingen J. Excessive bleeding in the floor of the mouth after endosseus implant placement: a report of two cases. Int J Oral Maxillofac Surg 2010;39:412-5.

21. Wasson M, Ghodke B, Dillon JK. Exsanguinating hemorrhage following third molar extraction: report of a case and discussion of materials and methods in selective embolization. $\mathrm{J}$ Oral Maxillofac Surg 2012;70:2271-5.

22. Bratton TA, Jackson DC, Nkungula-Howlett T, Williams CW, Bennett CR. Management of complex multi-space odontogenic infections. J Tenn Dent Assoc 2002;82:39-47.

23. Petitpas F, Mateo J, Blancal J-P, Mimoz 0. Fasciites cervicales nécrosantes. Prat Anesth Reanim 2010;14:4-8.

24. Yoshii T, Hamamoto Y, Muraoka S, Kohjitani A, Teranobu 0, Furudoi S, Komori T. Incidence of deep fascial space infection after surgical removal of the mandibular third molars. J Infect Chemother 2001;7:55-7.

25. Tran Ba Huy P, Blancal J-P, Verillaud B, Mebazaa A, Herman P. Cervico-facial fascitiis. A major ENT emergency. Bull Acad Natle Med 2011;195:661-76, discussion 676-8.

26. Petitpas F, Blancal J-P, Mateo J, Farhat I, Naija W, Porcher R, Beigelman C, Boudiaf M, Payen D, Herman P, Mebazaa A. Factors associated with the mediastinal spread of cervical necrotizing fasciitis. Ann Thorac Surg 2012;93:234-8.

27. Albilia JB, Humber CC, Clokie CML, Sándor GKB. Lemierre syndrome from an odontogenic source: a review for dentists. J Can Dent Assoc 2010;76:a47.

28. Chueng K, Clinkard DJ, Enepekides D, Peerbaye Y, Lin VYW. An unusual presentation of Ludwig's angina complicated by cervical necrotizing fasciitis: a case report and review of the literature. Case Rep Otolaryngol 2012;2012:931350.

29. Bilbault P, Castelain V, Schenck-Dhif M, Schneider F, Charpiot A. Life-threatening cervical necrotizing fasciitis after a common dental extraction. Am J Emerg Med 2008;26:971.e5-7.

30. Costain N, Marrie TJ. Ludwig's angina. Am J Med 2011;124:115-7. 